\section{Manufacturer Training of Electron Microscopy and Analysis Techniques}

\author{
Neil Rowlands \\ Oxford Instruments, Concord, MA \\ neil.rowlands@oxinst.com
}

Note: This article is based on a presentation at the Albuquerque M\&M meeting, held in August 2008. The presentation was one of 15 invited and contributed talks in a symposium entitled 'Teaching of Microscopy and Microanalysis' chaired by Elaine Schumacher and Charles Lyman. This issue of Microscopy Today includes three other articles derived from talks at that symposium, and future issues are expected to contain additional articles on this topic.

\section{Introduction}

Training by an instrument manufacturer is provided at various levels, ranging from basic operation requirements of instrumentation to high level courses for specific advanced level applications and integrated solutions.

Initial training usually is in the form of an initial familiarization performed by the installation engineer, and subsequent training is given at training courses at the instrument company laboratory or, in some instances, at the customer site. Manufacturer training can be required on different levels from initiation to basic techniques and operation of the instrumentation to advanced courses for specific applications. Although basic theory is present at the start of a course, the overall aim is to provide practical expertise on an operational level. It is encouraged that customers who are new to the techniques presented may avail themselves of more theoretically orientated courses available at universities and other private institutes.

\section{Initial familiarization}

This is an integral part of customer training, in that it is useful for the customer to have some prior knowledge of the instrumentation before attending a training course. This preliminary instruction is provided by the installation engineer in most cases. However, when dealing with complex equipment such as analytical TEMs it may be necessary that an applications specialist provide the initial familiarization with the principal user and in this case it is highly likely that the user will then take multiple training courses, (TEM, EELS, EDS) after the initial familiarization period has been completed. It is always recommended that the customer spend at least a month using the instrument directly after installation. This gives a comfort level that is often necessary to participate in a subsequent course fully.

\section{Training at the manufacturer's facility}

This is usually the preferred method of instrument training as it guarantees that all equipment will be optimized for the course. A number of experts will be available, and the likelihood of interruption by colleagues and customers is minimized (the use of mobile phones, PDA's etc. is generally discouraged in training classes). Courses are normally limited to about 5 or 6 people per class. The general structure of such course usually involves half a day to a day on basic theory and a description of the design and functionality of the instrumentation. The pre-knowledge of customers may vary widely and often the depth of this section may need to be tailored to the needs of the group.

The second and subsequent days of the course are of a more practical nature with hands-on instruction and exercises that may be performed at the instrument or at remote workstations. The ability to network the electron microscope and analytical systems to remote computers has proved most useful in demonstrating certain features of the instrumentation, especially, in tasks that may easily be performed off-line. This would include tasks such as post processing of EDS spectrum maps, in-depth analysis of EBSD or CBED patterns, and image analysis and particle characterization. More advanced courses may be offered in conjunction with the basic course. For instance, a course on basic EDS may be followed by a particle analysis course or a wavelength dispersive analysis course. Customers may choose to take these courses sequentially or, depending upon skill level, may wish to take the basics and then return at a later date for more specific and advanced courses. It is often advised that novice customers may want to postpone taking a specialized course until they have spent some time with the basic EDS course.

In the case of EBSD training, there is a far more intensive theoretical introduction as many new students are new to the field and lack the basic concepts of a technique that requires at least a working knowledge of crystallography and texture analysis. The classroom sessions are generally spread throughout the week in conjunction with practical sessions whereas the EDS classroom session is limited to the first day.

Courses for wavelength spectrometry are performed directly after the three-day EDS course and students may take it in conjunction with the EDS or separately depending upon the configuration and purchase period of each analytical technique. This is also the case with feature analysis courses.

Likewise, in the case of an SEM with analytical capability, it would be better to attain mastery of the operational aspects of the microscope before attending an analytical course as the configuration of the SEM may affect the analytical capabilities greatly. Therefore, the ability to

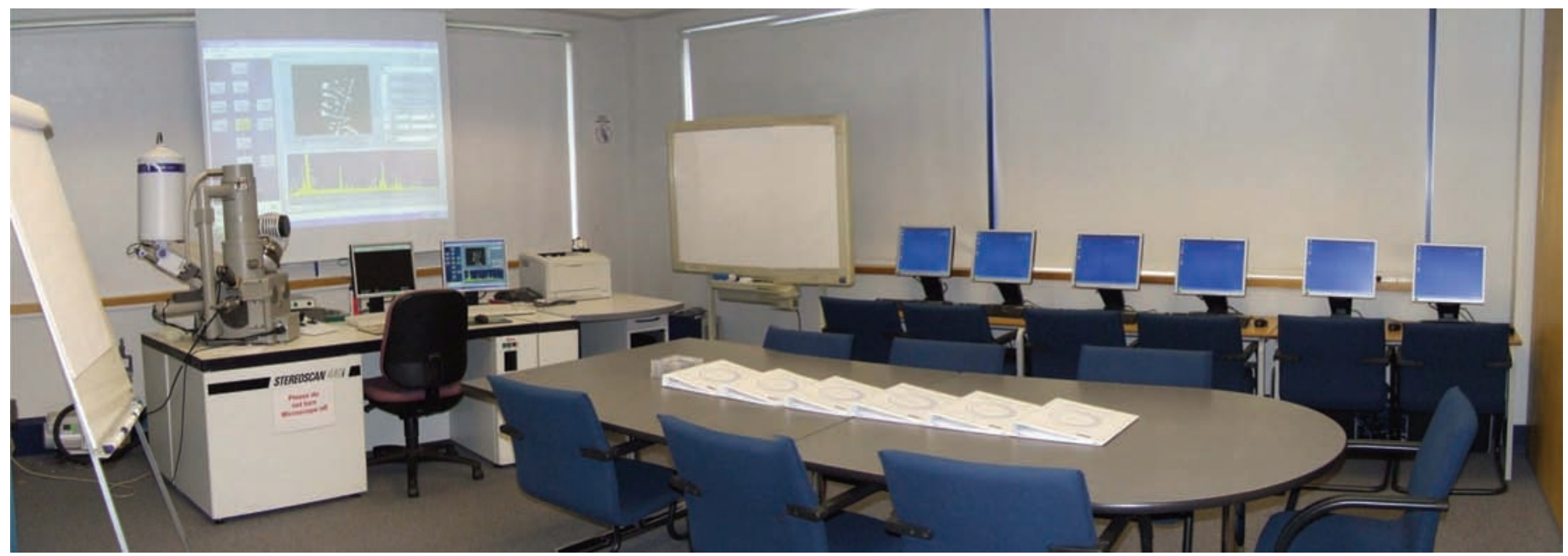

Figure 1. Typical manufacturer training facility 


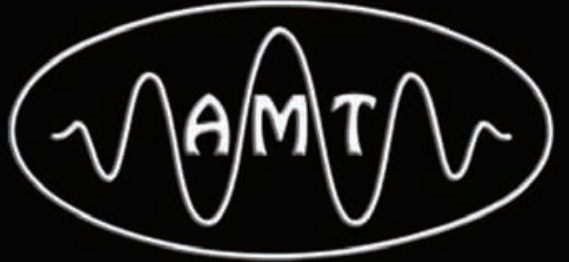

High Definition Digital TEM Cameras with

1 to 16 Megapixels
- AMT SOLUTIONS

- Life Science Cameras

- Material Science Cameras

- Easy To Use Software

- Reliability and Services

- TEM Integration

- Extensive Support
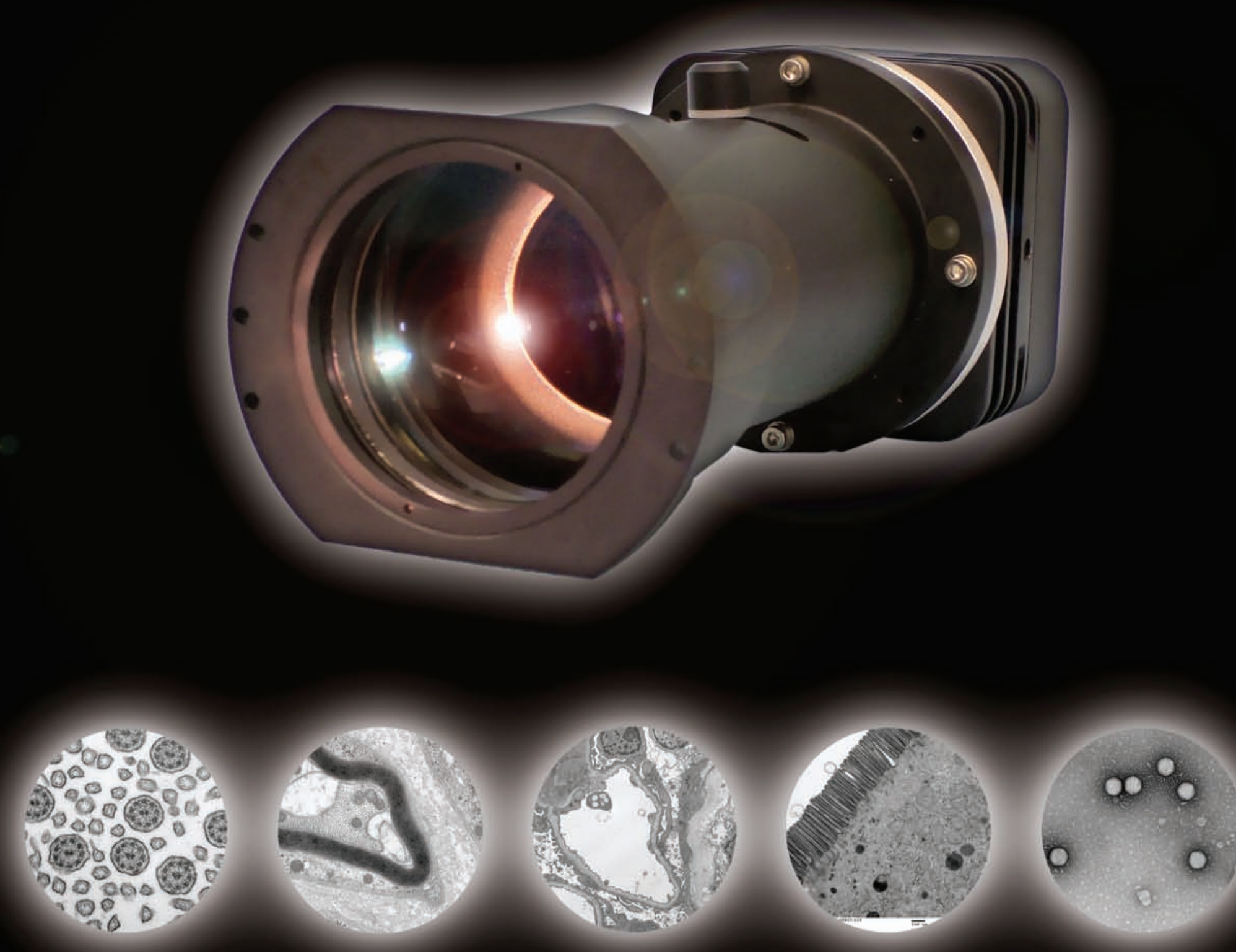
operate the SEM under a range of different conditions is usually necessary for attaining the best analytical results combined with optimum imaging conditions.

The ability to operate over a range of different excitation voltages is important, not just for imaging but also for improving spatial resolution in EDS and WDS, which is effected by using lower excitation voltages. However, spectral interpretation becomes more complex and training accommodates the theory and practice of such analyses. This is important because in the past, it has been generally accepted that analysis be performed at higher $\mathrm{kVs}$, but new Schottky field emission SEMs with higher beam currents and small probe sizes have made the use of low-kV data practical. Issues such as this indicate the value of attending training courses, as there are many developments that even seasoned SEM users may not be aware.

The conceptual approach to such courses is geared towards the competent use of the equipment and the understanding of the full capabilities of the hardware and software available. It is often found that customers who do not avail themselves of such courses do not utilize features that are present in the system because they did not realize that these features were included in their package. An added bonus to these training courses is that customers from different fields and industries have the ability to meet one another and compare samples and techniques.

\section{On-site training}

In general, training courses are performed at the manufacturer's facility, but may also be given at a customer site. On-site training may be necessary for TEM systems, for instance, when there is no equivalent instrument at the manufacturer's facility. On-site training may also be appropriate for specialized applications such as mineral liberation analysis and gunshot residue analysis and where a considerable number of people need to be trained who work on different shifts. This type of training is often the case in the semiconductor industry.

\section{TEM training}

A modern analytical TEM is a very complex analytical tool. The basic instrument is generally capable of high resolution imaging, generating various types of diffraction patterns and has the ability to perform various analytical tasks such as EDS, EELS, and sometimes energy filtering built into the column. Add aberration correctors and the depth of knowledge to operate such an instrument competently becomes considerable.

Training for large installations of this type does require prior knowledge of at least basic physics, but previous training is always desirable. In such a case, on-site training requires teaching the operator the basic techniques of operation. In other words, it is necessary to show basic alignments, conditions for high resolution, electron diffraction, EDS and EELS analysis. It is recommended that after such training the operators avail themselves of courses provided by the EDS and EELS manufacturers and also take some more theoretically based courses that are given at institutions such as Lehigh University short courses and the McCrone College of Microscopy.

\section{Advanced SEM applications}

It may be the case that a customer needs to use a specific technique that relies on a customized setup of both the SEM and the analytical system. Very often, this is instrument dependent and it is difficult to teach all the subtleties involved in a general course. This may include setting up certain parameters, such as stage movement and gray level adjustments that vary from instrument to instrument. Gun shot residue analysis is a case in point. It is essential that the system be set up to detect the maximum number of particles with the greatest accuracy at the fastest speed possible. In such cases, it is necessary for the opera- tor to work closely with the applications specialist. On-site training is usually essential in such circumstances to ensure the combined system is used to its best efficiency.

\section{On-site training for multiple users}

In many large industrial sites, such as semiconductor fabrication plants, there are multiple users and maybe multiple instruments, and it is not feasible to send all users to a manufacturer's training course. The economy of bringing a trainer onsite and performing a number of sessions outweighs the usual disadvantages inherent with training at a customer site. Usually this involves different shifts and although these operators only receive basic training, their supervisors, or the engineers may be able to attend more, in depth training courses later. Generally, these operators will be performing relatively basic tasks whereas data interpretation will be performed by the engineers. That being said, it is still of vital importance that the operator fully understands the underlying concepts of the techniques they will be putting to use.

\section{Remote training}

With increases in technology the demand for tele-presence training becomes more and more viable. With increased bandwidth it is now possible to run instruments remotely and to give multi-media presentations over the web in the same way as professors often simultaneously teach large classes in a number of lecture theaters. While this may not be the preferred method for replacing a traditional training course, it certainly can work for a short refresher course or a seminar on specific techniques. To be able to work directly on-screen with the customer rather than trying to explain details over a telephone line can be a great advantage where distances are great or the length of the training would not warrant a visit to the manufacturer's laboratory. With the increase in travel costs and lack of training time available, online training has increased in popularity. There is also increasing use of web-based tutorials or "webinars," which provide a forum for tackling individual or multiple topics in an interactive environment over the web. Usually, a select group with similar applications are chosen, depending upon the needs of the group and they can watch the presentations and interact with the speakers. It is even possible for the attendee to take control of the instrument to demonstrate a point. This can be further extended to web based seminars and tutorials that may be useful for those who do not have easy access to the manufacturer's facility.

\section{Online help}

It is now quite common to have online help included with an instrument. Some of these programs may be very sophisticated and offer interactive "bubble help" and tutorials during operation. The tutorials and other educational software may also be viewed off-line, so as not to tie up the instrument unduly.

\section{Conclusions}

Expert training from manufacturers is an indispensable part of running and maintaining analytical equipment such as SEMs, TEMs, and analytical equipment. Failure to attend such courses can lead to, at best, sub-optimal utilization of equipment and, at worst, obtaining the wrong results! In addition, it is highly likely that the full capabilities of the instrument may not be utilized due to aspects of hardware and software that are not immediately evident to those who have not taken a course.

It is always recommended that at least one person in a laboratory or institution should take a course of some kind and that person will then be able to pass this knowledge on to others in the group. New webbased software has drastically reduced the costs of training and allows for more flexibility for both the trainer and trainee. No excuse can be made for not receiving training. No matter how much experience one may have there is always something new to learn! 
Mini-SEM Compact Footprint $(22 \times 22 \times 22$ inches $)$

$15 \mathrm{keV}$ or $30 \mathrm{keV}$ Analytical Miniature Electron Microscope

120,000 X - Magnification (Digital Zoom)

Liquid Nitrogen Free - Light Element X-ray Detector

Easy Spectral Identification

Advanced Image Analysis \& Image Processing

Ultra Fast Elemental Mapping - Spectra for every pixel

Auto Report Writing

Installation \& Training in Minutes!

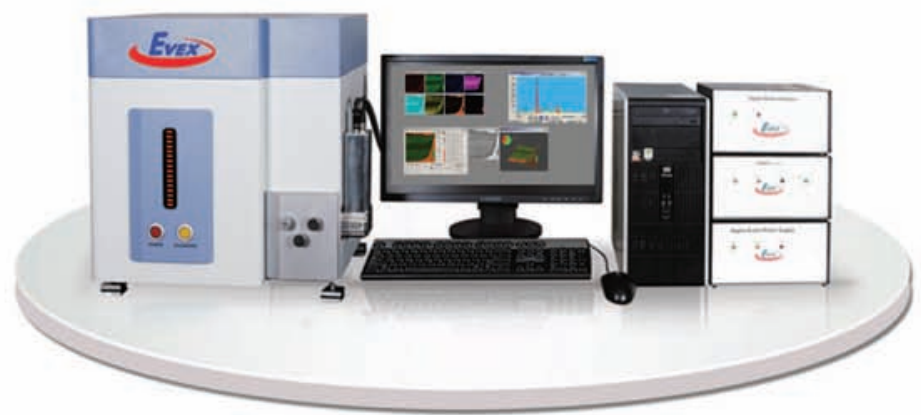

Scanning Electron Microscope $+$

X-ray NanoAnalysis

EVEX

Call Today

609-252-9192

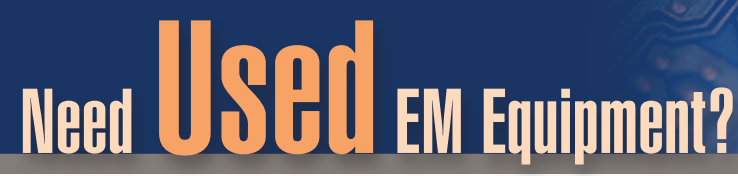

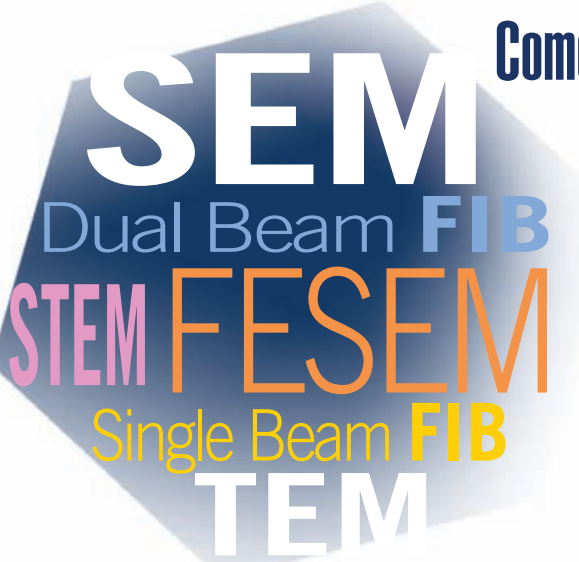

Whether you're looking to buy used EM equipment or have used equipment to sell, Angstrom Scientific is your source.

- Turnkey installation that is guaranteed operational

- All major SEM, TEM, STEM and FIB brands

- 40 years of experience and market knowledge

See our current inventory of used EM equipment at www.angstrom.us

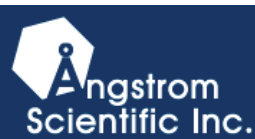

Angstrom Scientific, Inc.

(201)-760-2524

Info@angstrom.us

www.angstrom.us

\section{PELCO $^{\circledR}$ \\ Silicon Nitride Membranes \\ Next Generation $\mathrm{Si}_{3} \mathrm{~N}_{4}$ TEM Support Films}

- Durable and inert planar 50 and $200 \mathrm{~nm}$ substrates

- $3.0 \mathrm{~mm}$ circular frame

- EasyGrip ${ }^{\mathrm{TM}}$ edges

- Free from debris

- Complimented with Holey Membranes and Silicon Dioxide Substrates

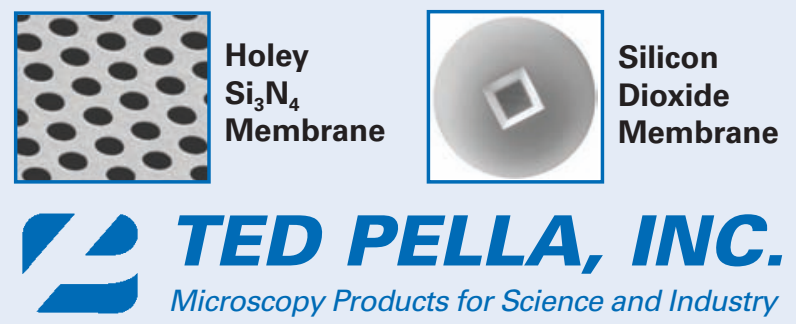

sales@tedpella.com 800-237-3526 www.tedpella.com 